\title{
COMPORTAMIENTO DE HÍBRIDOS DE MAÍZ Y APTITUD COMBINATORIA DE LÍNEAS CON CARACTERES CONTRASTANTES1
}

\author{
Narciso Vergara ${ }^{2}$, Shivaji Pandey ${ }^{3}$, Surinder Kumar ${ }^{3}$, Scott D. McLean ${ }^{3}$, Sergio Rodríguez ${ }^{2}$
}

\begin{abstract}
RESUMEN
Comportamiento de híbridos de maíz y aptitud combinatoria de líneas con caracteres contrastantes. Se identificaron doce líneas con mazorca larga y doce líneas con mazorca gruesa. Las cruzas simples fueron evaluadas bajo un diseño de látice simple 12 x 12 en cinco ambientes duran-te 1995. Estimaciones de aptitud combinatoria general (ACG) y específica (ACE) para rendimiento de grano se calcularon con análisis de línea x probador. La línea Pob.21xPob.43 registró el valor más alto de ACG $(0,58$ t/ha) dentro de líneas con mazorca larga, mientras que en el grupo de líneas con mazorca gruesa el valor más alto de ACG $(0,48 \mathrm{t} / \mathrm{ha})$ fue para la línea 19 (Pob.25). El más alto rendimiento $(8,35 \mathrm{t} / \mathrm{ha})$ fue para la cruza 4 x 22 (Pob.21xPob.43) x (Pob.32). Se muestra la importancia de considerar el uso de líneas con caracteres contrastantes y su patrón heterótico en el desarrollo de híbridos para maximizar el comportamiento de la Fl.
\end{abstract}

\begin{abstract}
Performance of coro hybrids and combining ability of inbred lines with different traits. Twelve long-eared and twelve thick-eared lines were identified for this purpose. Single crosses were evaluated using a 12 x 12 simple lattice design with four replications in five environments during 1995. General combining ability (GCA) and Specific combining ability (SCA) estimates for grain yield were calculated using line $x$ tester analysis. Line 4 (Pop.21xPop.43) registered the highest GCA value (0,58 t/ha) among long-eared lines; while in the group of thick-eared lines the highest GCA values $(0,48 \mathrm{t} / \mathrm{ha})$ corresponded to line 19 (Pop.25). The highest yield (8,35 t/ha) and SCA effect of $(0,61 \mathrm{t} / \mathrm{ha}$ ) was produced by cross 4 x 22 (Pop.21xPop.43) x (Pop.32). Results showed that it is important to consider the use of lines with different traits and their heterotic pattern to develop hybrids maximizing Fl performance.
\end{abstract}

\section{INTRODUCCIÓN}

En un programa de híbridos, la información de aptitud combinatoria de las fuentes de germoplasma (s) y de los progenitores derivados de ellas es un requerimiento importante, además de considerar su respuesta heterótica para incrementar la eficiencia en hibridación. Para obtener un nivel alto de heterosis debemos combinar progenitores endocriados y no endocriados o una combinación de ambos (Vasal et al., 1990), y además que pertenezcan a grupos heteróticos opuestos. Desde que en el CIMMYT se estableció un programa de hibridación en 1985, con la finalidad de inte.grar el mejoramiento poblacional con el desarrollo de híbridos, se han mejorado y desarrollado numerosas líneas tropicales y subtropicales algunas de las cuales son progenitoras de híbridos sobresalientes (Srinivasan et al., 1993). Todo este esfuerzo dio como resultado la liberación de 58 líneas tropicales y 42 subtropicales durante 1991-92. Mientras que en 1994 fueron anunciadas 35 líneas blancas y 27 líneas amarillas tropicales. El uso de semilla híbrida de cruza simple ha aumentado recientemente en los países en vías de desarrollo (CIMMYT, 1994), la razón de que se cultive este tipo de híbridos es que presentan mayor uniformidad y potencial de rendimiento, por lo tanto es importante desarrollar progenitores con buenos atriblltos agronómicos que faciliten la producción de semilla híbrida. Por los antecedentes expuestos,

\footnotetext{
1 Presentado en Ja XLIH Reunión Anual del PCCM CA en Panamá. 1997.

2 Universidad Autonoma Agraria Antonio Narro (UAAAN)

3 Centro Internacional de Mejoramiento de Maíz y Trigo (CIMMYT), Apdo. Postal 6-649, Col. Juarez, Delg. Cuahtemoc 06600.
} 
el presente trabajo se planteó para determinar si el uso de líneas endocriadas con caracteres contrastantes en tipo de mazorca permitiría identificar híbridos con buen potencial de rendimiento.

\section{MATERIALES Y MÉTODOS}

Durante el desarrollo de líneas en el programa de híbridos de CIMMYT, se identificaron y clasificaron varias líneas tropicales de acuerdo a su tipo de mazorca, se formaron dos grupos con doce líneas cada uno. El primero incluyó líneas con mazorca larga, derivadas principalmente de Población 21 y 43, y en el segundo se incluyeron líneas con mazorca gruesa derivadas de Población 22, 25, 32, Pool 24, Tuxpeño Sequía (Cuadro 1). Con estos dos grupos se formaron cruzas apareadas en forma de diseño II, se generaron 144 cruzas simples, y se evaluaron en ensayos de rendimiento en cinco localidades (Cuadro 2); tres bajo condiciones tropicales y dos subtropicales. Se utilizó un diseño experimental de látice 12 x 12 simple con cuatro repeticiones. En cada ensayo la unidad experimental consistió de un surco de cinco metros de largo, espaciado a $75 \mathrm{~cm}$ entre surcos y $25 \mathrm{~cm}$ entre plantas para obtener una densidad de 53.000 plantas por hectárea. Los 24 progenitores, se evaluaron en un ensayo separado durante 1996 ciclo A, en Poza Rica Veracruz. Debido a la poca semilla disponible, durante la conducción y desarrollo de los experimentos se evaluó floración femenina y masculina al $50 \%$, altura de planta y mazorca, acame de raíz y tallo, cobertura de mazorca y pudrición de mazorca antes de la cosecha. Para los datos de longitud, diámetro y número de hileras, se tomaron diez mazorcas al azar en cada parcela en el ensayo de líneas per se y en el ensayo de cruzas en Poza Rica y Tlaltizapan se registró el promedio para cada entrada respectivamente. Al momento de la cosecha se registró el peso fresco de mazorca y el por ciento de humedad de el grano. El rendimiento de grano en (t/ha) fue calculado por parcela, asumiendo el $80 \%$ de desgrane y ajustándolo al 15,5\%
Cuadro 1. Descripción de líneas de maíz blanco tropical de CIMMYT utilizadas en este estudio.

\begin{tabular}{lll}
\hline \multicolumn{2}{l}{ Líneas con mazorca larga } & \\
\hline 1 & Pob.21 C5 HC219-3-1-B-ff-\#\# & CML-9 \\
2 & Pob.21 C5 HC219-3-2-2-3-B-2-2-ff-\# & CML-10 \\
3 & Pob.21 C5 HC219-3-2-2-3-B-4-1-\#\# & \\
4 & (Pob.21 HC219 x Pob.43 HC95)-5-BBB-1-\#\#\# & \\
5 & (Pob.21 TSR-2 x Pob.21HC219)-4-BBB-2-\#-BBB & \\
6 & (AC7643 x Pob.43)-2-3-4-2-BBB-1-B-\#-BBB & \\
7 & (Ac7643 x Pob.43 HC7)-2-3-2-1-BB-f & CML-273 \\
8 & Pob.43 STE C1 HC10-2-1-1-1-3-BBB-1-\#\#-BBB & \\
9 & Pob.21 C5 HC219-3-1-\#\#-8-1-3-BBB-f & CML-264 \\
10 & AC7643-16-F3-\#-4-BBBB-\#\#-B & \\
11 & Pob.21 C6 S1 MH226-F3-\#-10-BBBBBBB & \\
12 & Pob.21 C5 HC84-F3-\#-5-BBBBBBB & \\
\hline Líneas con mazorca gruesa & \\
\hline & & \\
13 & (P24MH119 x MH54)-6-4-1-1-BB-f & CML-247 \\
14 & (P24MH119 x MH54)-6-3-2-1-BBB-2-B-\#-B & \\
15 & P24(S1)-2-B-3-B-\#\#-B & CML-39 \\
16 & Tuxp.Seq.149-2-BBB-\#-1-BB-f & \\
17 & Tuxp.Seq.219-3-4-2-B-\#\#-2-BBB-1-\#\#-BBBB & \\
18 & Pob.22 TSR-4-3-1-3-1-BBB-f & CML-267 \\
19 & Pob.25 C0 HC246-3-1-BB-2-\#\#-BB & \\
20 & Pob.25 STE C1 HC5-2-1-1-\#-BBBB-1-\#\#-B & \\
21 & Pob.32 C4 HC242-3-1-B-ff-\#\# & \\
22 & Pob.32(MRRS) C1-659-1-BBBBBBB & \\
23 & Sint. Bco. TSR-3-1-2-3-2-BBBB-\#-BBB & \\
24 & Pob.21 C5 HC109-3-1-5-4-B-4-3-\#\#-2-BBBBBB & \\
\hline & & \\
\hline & & \\
\hline
\end{tabular}

CML (Son líneas liberadas por CIMMYT).

de humedad. Se realizó un análisis de varianza línea $\mathrm{x}$ probador para cada localidad así como un análisis combinado para rendimiento de grano usando el promedio de cada cruza a través de repeticiones, con base en el método descrito por Kempthorne en 1957.

Cuadro 2. Ambientes donde los ensayos de 144 cruzas simples fueron evaluados durante 1995, México.

\begin{tabular}{llcccl}
\hline Localidad & & $\begin{array}{c}\text { Altura } \\
(\mathbf{m s n m})\end{array}$ & $\begin{array}{c}\text { Latitud } \\
(\mathbf{N})\end{array}$ & $\begin{array}{c}\text { Longitud } \\
(\mathbf{O})\end{array}$ & $\begin{array}{c}\text { Mega } \\
\text { Ambiente }\end{array}$ \\
\hline Cotaxtla, Ver & (CO) & 15 & $18^{\circ} 50^{`} \mathrm{~N}$ & $96^{\circ} 10^{\circ} \mathrm{O}$ & Tropical \\
Poza Rica, Ver & (PR) & 60 & $20^{\circ} 32^{`} \mathrm{~N}$ & $97^{\circ} 26^{\circ} \mathrm{O}$ & Tropical \\
Cardel, Ver & (CA) & 85 & $19^{\circ} 20^{`} \mathrm{~N}$ & $96^{\circ} 23^{`} \mathrm{O}$ & Tropical \\
Tlaltizapan, Mor & (TL) & 840 & $18^{\circ} 41^{`} \mathrm{~N}$ & $99^{\circ} 68^{\circ} \mathrm{O}$ & Subtropical \\
Gómez Farías, Jal & (GF) & 1575 & $20^{\circ} 28^{`} \mathrm{~N}$ & $10^{\circ} 27^{\circ} \mathrm{O}$ & Subtropical \\
\hline
\end{tabular}


Los efectos de aptitud combinatoria general (ACG) y específica (ACE) fueron calculados para rendimiento de grano. Las pruebas de f para el análisis de varianza combinado, fueron calculadas tomando en cuenta los efectos principales como líneas y probadores fueron probadas con sus respectivas interacciones con localidades, el resto de las interacciones se probaron contra la interacción de líneas x probadores x localidades. Mientras que los efectos de ACG y ACE se probaron usando una simple prueba de $t$, de acuerdo con las fórmulas de Singh; Choudhary (1985).

\section{RESULTADOS Y DISCUSIÓN}

Los resultados obtenidos por el análisis de varianza combinado línea $x$ probador se presentan en el Cuadro 3. Hubo diferencias altamente significativas en las fuentes de variación, excepto para líneas. Lo que indica que los materiales se comportaron de forma diferente en una localidad a otra. Estos resultados coinciden con los indicados por Pandey; Vargas (1985) donde muestran que los materiales gen éticos, mientras más seleccionados y más homocigotes, más interaccionan con el ambiente. También se observó que las líneas con mazorca gruesa en grupo dos, registraron valores más altos de ACG que las líneas con mazorca larga. Esto sugiere que se debe considerar caracteres contrastantes en tipo de mazorca durante le desarrollo de las líneas.

El rendimiento promedio de 144 cruzas simples por localidad se presentan en el Cuadro 4. En Tlaltizapan se registró el promedio más alto $(8,91$ t/ha), y en

Cuadro 3. Análisis de varianza líneas $\mathrm{x}$ provador combinado para rendimiento de grano $(\mathrm{kg}$ /ha) probado en cinco localidades en 1995, México.

\begin{tabular}{lrc}
\hline Fuentes de variación & GL & Cuadros medidos \\
\hline Localidades (Loc.) & 4 & $484070584^{* *}$ \\
Líneas' (AGG) $^{*}$ (11 & $5850854^{*}$ \\
Líneas x Loc. & 44 & $2386960^{* *}$ \\
Provadores ${ }^{2}$ (ACG) & 11 & $7049029^{* *}$ \\
Provadores x Loc. & 44 & $2374306^{* *}$ \\
Líneas x Provador (ACE) & 121 & $822269^{* *}$ \\
Líneas x Provador x Loc. & 484 & $306903^{* *}$ \\
Error combinado & 2160 & 205433 \\
\hline
\end{tabular}

* Diferencias significativas $\mathrm{p}=0,05$.

**Diferencias significativas $\mathrm{p}=0,01$.

1 Líneas con mazorca larga en grupo 1.

2 Líneas con mazorca gruesa en grupo 2.
Cuadro 4. Localidades y sus medidas de rendimiento de grano en (t/ha) de 144 cruzas simples. Verano, 1995. México.

\begin{tabular}{lccc}
\hline Localidad & $\begin{array}{c}\text { Rendimiento } \\
\text { de grano } \\
(\mathbf{t} / \mathbf{h a})\end{array}$ & $\begin{array}{c}\text { Coeficiente } \\
\text { de variación } \\
(\boldsymbol{\%})\end{array}$ & $\begin{array}{c}\text { Humedad } \\
\text { de grano } \\
(\boldsymbol{\%})\end{array}$ \\
\hline Cotaxtla, Veracruz & 8,05 & 15,5 & 26,0 \\
Poza Rica, Veracruz & 7,49 & 11,4 & 20,5 \\
Cardel, Veracruz & 5,51 & 12,6 & 22,5 \\
Tlaltizapan, Morelos & 8,91 & 8,6 & 23,4 \\
Gómez Farías, Jalisco & 4,49 & 19,2 & 21,2 \\
\hline Media general & 6,89 & & \\
\hline
\end{tabular}

Gómez Farías (4,9 t/ha) el más bajo, debido a que el germoplasma utilizado se encuentra mejor adaptado en la primera. El coeficiente de variación estuvo entre $8,6 \%$ a $19,2 \%$ y una humedad de grano de $20,5 \%$ a $26,0 \%$. La media general registrada fue de 6,89 t/ha.

Doce de las 24 líneas mostraron efectos positivos de ACG para rendimiento (Cuadro 5). La línea cuatro tuvo el valor más alto de ACG $(0,58 \mathrm{t} / \mathrm{ha})$ dentro del grupo de líneas con mazorca larga y un comportamiento en cruzamiento de $7,47 \mathrm{t} / \mathrm{ha}$, mostrando su estabilidad para ACG ya que registró valores positivos en cuatro de las cinco localidades. La línea 9 ( $\mathrm{ACG}=0,32 \mathrm{t} / \mathrm{ha}) \mathrm{re}$ gistró efectos positivos en las cinco localidades, esta línea fue liberada en 1994. Dentro del grupo de líneas con mazorca gruesa, la línea 19 fue la única con alto valor de $\mathrm{ACG}=0,48 \mathrm{t} / \mathrm{ha}$ y un rendimiento promedio de 7,37/ha y una buena estabilidad a través de ambientes.

En el Cuadro 6, se muestra el comportamiento de 24 líneas per se y en cruzamiento. Dentro de las líneas con mazorca larga el rendimiento más alto fue registrado por la línea $8(4,35 \mathrm{t} / \mathrm{ha})$ seguido por la línea $7(4,13$ t/ha) estas dos líneas provienen de Población 43 (La Posta). En el grupo de líneas con mazorca gruesa, la línea 23 registró el más alto rendimiento $(4,36 \mathrm{t} / \mathrm{ha})$ seguido por la línea 15 (4,02 t/ha). Los datos de longitud, diámetro y número de hileras registrados en cada grupo de líneas, muestran que la clasificación en tipo de mazorca fue correcta. Donde las líneas con mazorca larga registraron valores más altos para longitud de mazorca, mientras que en las líneas con mazorca gruesa, los valores de diámetro y número de hileras fueron más importantes. Sin embargo analizando el comportamiento en cruzamiento de cada grupo de líneas para rendimiento y caracteres de mazorca, se observó un rendimiento ligeramente mayor para las cruzas en el segundo grupo así como también para diámetro de mazorca, no ocurriendo así para longitud y número de hileras de mazorca. 
Cuadro 5. Aptitud combinatoria general y rendimiento promedio en (t/ha) para 12 líneas que mostraron efectos positivos de ACG, através de cinco localidades, México.

\begin{tabular}{|c|c|c|c|c|c|c|c|}
\hline Líneas (grupo 1) & ACG & EST. $^{2}$ & Rend. 1 & Líneas (grupo 2) & ACG & EST.2 & Rend.1 \\
\hline 4 & 0,58 & $5(4)$ & 7,47 & 19 & 0,48 & $5(4)$ & 7,37 \\
\hline 9 & 0,32 & $5(5)$ & 7,21 & 23 & 0,30 & $5(4)$ & 7,19 \\
\hline 7 & 0,17 & $5(3)$ & 7,06 & 16 & 0,28 & $5(3)$ & 7,17 \\
\hline 3 & 0,14 & $5(2)$ & 7,03 & 22 & 0,27 & $5(5)$ & 7,16 \\
\hline 5 & 0,03 & $5(2)$ & 6,92 & 24 & 0,23 & $5(2)$ & 7,12 \\
\hline 11 & 0,02 & $5(3)$ & 6,91 & 20 & 0,08 & $5(4)$ & 6,97 \\
\hline
\end{tabular}

* Significancia al 0,05 nivel de probabilidad.

** Significancia al 0,01 nivel de probabilidad.

1 Rendimiento de grano ( $\mathrm{t} / \mathrm{ha}$ ) de cada linea promediadas sobre 12 probadores.

2 Estabilidad de ACG (valores positivos de Acg en cada localidad).

Cuadro 6. Rendimiento de grano (t/ha) per se y comportamiento en cruzamiento de 24 líneas y longitud, diámetro y número de hileras de mazorca, México.

\begin{tabular}{|c|c|c|c|c|c|c|c|c|}
\hline $\begin{array}{l}\text { Líneas } \\
\text { Grupo } 1\end{array}$ & $\begin{array}{l}\text { Rend. }^{1} \\
\text { (t/ha) }\end{array}$ & $\begin{array}{l}\text { Long. }{ }^{1} \\
(\mathrm{~cm})\end{array}$ & $\begin{array}{l}\text { Diam. }{ }^{1} \\
(\mathrm{~cm})\end{array}$ & Hileras' & $\begin{array}{l}\text { Rend. }^{2} \\
\text { (t/ha) }\end{array}$ & $\begin{array}{l}\text { Long. }^{3} \\
\text { (cm) }\end{array}$ & $\begin{array}{c}\text { Diam. }^{3} \\
(\mathrm{~cm})\end{array}$ & Hileras. $^{3}$ \\
\hline 1 & 3,97 & 12,3 & 3,8 & 14 & 6,89 & 15,6 & 4,7 & 165 \\
\hline 2 & 3,45 & 15,6 & 3,2 & 10 & 6,77 & 17,4 & 4,4 & 14 \\
\hline 3 & 3,95 & 16,9 & 3,5 & 10 & 7,03 & 17,6 & 4,5 & 15 \\
\hline 4 & 3,14 & 15,0 & 3,8 & 14 & 7,47 & 16,6 & 4,8 & 17 \\
\hline 5 & 2,8 & 17,9 & 3,2 & 12 & 6,92 & 16,9 & 4,4 & 15 \\
\hline 6 & 3,57 & 12,9 & 3,9 & 14 & 6,84 & 15,6 & 4,7 & 16 \\
\hline 7 & 4,13 & 12,4 & 4,0 & 14 & 7,06 & 15,7 & 4,7 & 16 \\
\hline 8 & 4,35 & 14,6 & 3,6 & 12 & 6,61 & 15,9 & 4,6 & 15 \\
\hline 9 & 2,68 & 10,8 & 3,8 & 14 & 7,21 & 15,0 & 4,8 & 16 \\
\hline 10 & 3,51 & 15,1 & 3,8 & 12 & 6,19 & 15,3 & 4,4 & 14 \\
\hline 11 & 2,76 & 11,1 & 4,1 & 16 & 6,91 & 15,8 & 4,7 & 17 \\
\hline 12 & 3,28 & 12,6 & 3,6 & 12 & 6,79 & 16,1 & 4,5 & 15 \\
\hline Media & 3,47 & 13,9 & 3,7 & 13 & 6,89 & 16,2 & 4,6 & 15 \\
\hline Máximo & 4,35 & 17,9 & 4,1 & 16 & 7,47 & 17,6 & 4,8 & 17 \\
\hline Mínimo & 2,68 & 10,8 & 3,2 & 10 & 6,19 & 15,0 & 4,4 & 14 \\
\hline $\begin{array}{l}\text { Líneas } \\
\text { Grupo } 2\end{array}$ & $\begin{array}{l}\text { Rend, }{ }^{1} \\
(\mathrm{t} / \mathrm{ha})\end{array}$ & $\begin{array}{l}\text { Long, }{ }^{\prime} \\
\text { (cm) }\end{array}$ & $\begin{array}{l}\text { Diam. }^{1} \\
(\mathrm{~cm})\end{array}$ & Hileras.' & $\begin{array}{l}\text { Rend.* } \\
\text { (t/ha) }\end{array}$ & $\begin{array}{l}\text { Long. }^{3} \\
\text { (cm) }\end{array}$ & $\begin{array}{l}\text { Diam. }^{3} \\
(\mathrm{~cm})\end{array}$ & Hileras. $^{3}$ \\
\hline 13 & 3,49 & 11,5 & 4,2 & 14 & 6,65 & 15,5 & 4,6 & 16 \\
\hline 14 & 3,21 & 11,9 & 4,4 & 18 & 6,88 & 15,9 & 4,7 & 17 \\
\hline 15 & 4,02 & 14,6 & 4,1 & 14 & 6,81 & 17,9 & 4,5 & 15 \\
\hline 16 & 2,73 & 11,5 & 3,5 & 10 & 7,17 & 16,5 & 4,5 & 14 \\
\hline 17 & 2,52 & 10,2 & 4,0 & 14 & 6,51 & 15,4 & 4,6 & 16 \\
\hline 18 & 2,61 & 12,2 & 4,0 & 14 & 6,69 & 16,3 & 4,6 & 16 \\
\hline 19 & 2,91 & 10,0 & 4,0 & 16 & 7,37 & 16,1 & 4,6 & 16 \\
\hline 20 & 2,34 & 8,5 & 4,2 & 18 & 6,97 & 15,3 & 4,7 & 17 \\
\hline 21 & 3,27 & 12,3 & 4,4 & 16 & 6,18 & 15,5 & 4,6 & 16 \\
\hline 22 & 2,27 & 13,2 & 3,5 & 14 & 7,16 & 17,2 & 4,5 & 15 \\
\hline 23 & 4,36 & 13,3 & 4,0 & 14 & 7,19 & 16,5 & 4,6 & 15 \\
\hline 24 & 3,23 & 10,8 & 4,1 & 12 & 7,12 & 16,3 & 4,7 & 15 \\
\hline Media & 3,08 & 11,7 & 4,0 & 15 & 6,89 & 16,2 & 4,6 & 15 \\
\hline Máximo & 4,36 & 14,6 & 4,4 & 18 & 7,37 & 17,9 & 4,7 & 17 \\
\hline Mínimo & 2,27 & 8,5 & 3,5 & 10 & 6,18 & 15,3 & 4,5 & 14 \\
\hline
\end{tabular}

' Datos de una localidad (Poza Rica 96 A).

${ }^{2}$ Promedio de cada línea sobre doce líneas con mazorca gruesa en cinco ambientes

${ }^{3}$ Datos de dos localidades (Poza Rica y Tlaltizapan 95 B).

* Promedio de cada línea sobre doce líneas con mazorca larga en cinco ambientes. 
El comportamiento de cruzas simples junto con sus contribuciones de ACG y ACE se presentan en el Cuadro 7. Tres de la mejores cruzas obtuvieron buenos rendimientos y valores de ACE aceptables. La cruza 4 x 22 registró un rendimiento de 8,35 tJha, involucrando dos líneas con buena ACG.

La línea cuatro intervino en tres de las mejores cruzas, siendo una de las líneas más estables para ACG. En general las líneas con buenos efectos de ACG en sus respectivas combinaciones formaron buenas cruzas, ocurriendo lo contrario en las cruzas inferiores en rendimiento.

Cuadro 7. Comportamiento promedio de cinco cruzas superiores y cinco cruzas inferiores junto con sus contribuciones de ACG y ACE, a través de cinco localidades. Verano, 95. México.

\begin{tabular}{|c|c|c|c|c|c|}
\hline \multirow[t]{2}{*}{ Cruzas } & \multirow[t]{2}{*}{ Germoplasma } & \multirow{2}{*}{$\begin{array}{l}\text { Rend } \\
\text { (t/ha) }\end{array}$} & \multicolumn{2}{|c|}{ ACG } & \multirow[b]{2}{*}{ ACE } \\
\hline & & & P1 & $\mathbf{P 2}$ & \\
\hline $4 \times 22$ & Pob. $21 * 43 \times$ Pob. 32 & 8,35 & 0,58 & 0,27 & 0,61 \\
\hline $4 \times 24$ & Pob. $21 * 43 \times$ Pob. 21 & 8,26 & 0,58 & 0,23 & 0,56 \\
\hline $9 \times 24$ & Pob.21 x Pob.21 & 8,09 & 0,32 & 0,23 & 0,66 \\
\hline $9 \times 19$ & Pob.21 x Pob. 25 & 7,94 & 0,32 & 0,48 & 0,26 \\
\hline $4 \times 19$ & Pob. $21 * 43 \times$ Pob. 25 & 7,68 & 0,58 & 0,48 & $-0,27$ \\
\hline $9 \times 21$ & Pob.21 x Pob.32 & 5,71 & 0,32 & $-0,71$ & $-0,78$ \\
\hline $8 \times 21$ & Pob.43 x Pob.32 & 5,69 & $-0,28$ & $-0,71$ & $-0,21$ \\
\hline $10 \times 21$ & AC7643x Pob. 32 & 5,48 & $-0,70$ & $-0,71$ & $-0,00$ \\
\hline $10 \times 14$ & AC7643x Pob. $24 * 24$ & 5,41 & $-0,70$ & $-0,01$ & $-0,78$ \\
\hline $10 \times 13$ & AC7643x Pob. $24 * 24$ & 5,38 & $-0,70$ & $-0,25$ & $-0,57$ \\
\hline Promedio & & 6,89 & & & \\
\hline
\end{tabular}

El Cuadro 8, muestra el comportamiento agronómico de cruzas superiores e inferiores. Las cruzas superiores fueron un poco más precoces y con más altura de planta y mazorca, registrando también mazorcas más largas, más gruesas y con mayor número de hileras en comparación con las cruzas de bajo rendimiento. Dentro de las cruzas simples con mayor rendimiento (Cuadro 9), se observó que la línea 19 (Pob.25) se involucróen cuatro de las mejores diez cruzas, y tuvo buena ACG. La superioridad en rendimiento fue de $12,3 \%$ a $21,2 \%$ con respecto a la media general. En general se observaron buenas combinaciones entre Población $21 \mathrm{x}$ Población 25 y Población 21 x Población 32, este último un patrón heterótico y ampliamente conocido (Vasal, et al, 1986). Sin embargo algunas combinaciones entre líneas derivadas del mismo germoplasma como es el caso de 9x24, 1×24 y 4x24, las cuales involucran líneas derivadas de Población 21, pueden ser una opción para formar buenos híbridos. Estos resultados muestran que es posible formar híbridos interlineales intrapoblacionales con buen potencial de rendimiento, coincidiendo con lo reportado por Vasal, et al 1993.

Las cruzas superiores en rendimiento en cada localidad se presentan en el Cuadro 10. Se observó la misma tendencia, las mejores combinaciones involucraron líneas de Población 21 y líneas de Población 25, y Población 32. Estos resultados muestran híbridos con buenas características agronómicas como tipo de planta y posición de mazorca aceptable, así como resistencia al acame de raíz y tallo, además de mazorcas sanas y buenos atributos en tipo de mazorca.

Cuadro 8. Rendimiento de grano, días a floración femenina, altura de planta, altura de mazorca de cinco cruzas superiores y cinco inferiores junto con longitud, diámetro y número de hileras de mazorca, a través de cinco ambientes. Verano, 95. México.

\begin{tabular}{|c|c|c|c|c|c|c|c|c|}
\hline Cruzas & Germoplasma & $\begin{array}{l}\text { Rend } \\
\text { (t/ha) }\end{array}$ & $\begin{array}{c}\text { Floración } \\
\text { (días) }\end{array}$ & $\begin{array}{c}\text { A/Planta } \\
\text { (cm) }\end{array}$ & $\begin{array}{c}\text { A/Mazorca } \\
(\mathbf{c m})\end{array}$ & $\begin{array}{l}\text { Long. }^{3} \\
\text { (cm) }\end{array}$ & $\begin{array}{c}\text { Diam. }^{3} \\
(\mathrm{~cm})\end{array}$ & Hileras \\
\hline $4 \times 22$ & Pob. $21 * 43 \times$ Pob. 32 & 8,35 & 65 & 255 & 125 & 17,6 & 4,6 & 16 \\
\hline $4 \times 24$ & Pob. $21 * 43 \times$ Pob. 21 & 8,26 & 65 & 260 & 133 & 17,1 & 5,2 & 16 \\
\hline $9 \times 24$ & Pob.21 x Pob.21 & 8,09 & 66 & 252 & 130 & 14,6 & 4,9 & 16 \\
\hline $9 \times 19$ & Pob.21 x Pob. 25 & 7,94 & 65 & 250 & 130 & 15,3 & 4,8 & 18 \\
\hline $11 \times 22$ & Pob.21 x Pob. 32 & 7,89 & 66 & 243 & 131 & 16,6 & 4,7 & 16 \\
\hline $9 \times 21$ & Pob.21 x Pob.32 & 5,71 & 66 & 226 & 111 & 13,9 & 4,9 & 16 \\
\hline $8 \times 21$ & Pob.43 x Pob.32 & 5,69 & 65 & 238 & 121 & 14,4 & 4,6 & 16 \\
\hline $10 \times 21$ & AC7643 x Pob.32 & 5,48 & 66 & 227 & 115 & 15,6 & 4,3 & 14 \\
\hline $10 \times 14$ & AC7643 x Pob. $24 * 24$ & 5,41 & 67 & 219 & 102 & 15,4 & 4,4 & 16 \\
\hline $10 \times 13$ & AC7643 x Pob. $24 * 24$ & 5,38 & 65 & 216 & 110 & 15,7 & 4,2 & 14 \\
\hline Promedio & & 6,89 & 65 & 238 & 125 & 15,6 & 4,5 & 15 \\
\hline Máximo & & 8,35 & 68 & 267 & 146 & 20,9 & 5,2 & 18 \\
\hline Mínimo & & 5,38 & 62 & 212 & 102 & 13,7 & 4,1 & 12 \\
\hline
\end{tabular}


Cuadro 9. Rendimiento de grano, y comportamiento agronómico a través de localidades de híbridos de cruza simple superiores Verano 95. México.

\begin{tabular}{|c|c|c|c|c|c|c|c|c|}
\hline \multirow[t]{2}{*}{ Cruzas } & \multirow[t]{2}{*}{ Germoplasma } & \multirow{2}{*}{$\begin{array}{l}\text { Rend } \\
(\mathbf{t} / \mathbf{h a})\end{array}$} & \multirow[t]{2}{*}{$(\%)^{1}$} & \multicolumn{5}{|c|}{ Ambientes individuales ( $t / h a)$} \\
\hline & & & & $\mathrm{CO}$ & PR & CA & TL & GF \\
\hline $4 \times 22$ & Pob. $21 * 43 \times$ Pob. 32 & 8,35 & 121,2 & 10,08 & 8,77 & 6,60 & 10,85 & 5,45 \\
\hline $4 \times 24$ & Pob. $21 * 43 \times$ Pob. 21 & 8,26 & 119,9 & 10,02 & 7,91 & 5,87 & 11,50 & 5,98 \\
\hline $9 \times 24$ & Pob.21 x Pob.21 & 8,09 & 117,4 & 9,27 & 8,71 & 5,63 & 9,41 & 7,44 \\
\hline $9 \times 19$ & Pob.21 x Pob. 25 & 7,94 & 115,2 & 9,10 & 9,70 & 7,00 & 9,37 & 4,53 \\
\hline $11 \times 22$ & Pob.21 x Pob. 32 & 7,89 & 114,5 & 9,27 & 9,16 & 5,52 & 10,56 & 4,93 \\
\hline $1 \times 24$ & Pob.21 x Pob.21 & 7,87 & 114,2 & 10,30 & 7,87 & 5,91 & 9,51 & 5,75 \\
\hline $3 \times 19$ & Pob. 21 x Pob. 25 & 7,84 & 113,8 & 8,65 & 8,93 & 6,41 & 10,19 & 5,00 \\
\hline $1 \times 19$ & Pob. 21 x Pob.25 & 7,79 & 112,9 & 7,91 & 8,69 & 6,98 & 10,42 & 4,93 \\
\hline $5 \times 19$ & Pob.21*21 x Pob. 25 & 7,76 & 112,6 & 9,38 & 9,01 & 7,62 & 8,65 & 4,14 \\
\hline $9 \times 23$ & Pob.21 x Sint.Bco.Tsr & 7,74 & 112,3 & 8,59 & 8,26 & 6,14 & 10,46 & 5,26 \\
\hline Promedio & & 6,59 & & 8,05 & 7,49 & 5,51 & 8,91 & 4,49 \\
\hline
\end{tabular}

1 Superioridad sobre la media general.

Cuadro 10. Medias de rendimiento de grano (t/ha) y comportamiento agronómico de cruzas simples superiores en cada localidad. México, 1995.

\begin{tabular}{|c|c|c|c|c|c|c|c|c|c|c|c|c|c|}
\hline \multirow[t]{2}{*}{ Localidad } & \multirow[t]{2}{*}{ Cruzas } & \multirow[t]{2}{*}{ Germoplasma } & \multirow{2}{*}{$\begin{array}{l}\text { Rend } \\
\text { (t/ha) }\end{array}$} & \multirow{2}{*}{ (\%) 1} & \multirow{2}{*}{$\begin{array}{c}50 \% \\
\text { Estigm. }\end{array}$} & \multirow{2}{*}{$\begin{array}{l}\text { Altura } \\
\text {. Maz. }\end{array}$} & \multicolumn{2}{|c|}{ Acame } & \multirow{2}{*}{$\begin{array}{c}(\%) \\
\text { Maz. } \\
\text { Podrid. }\end{array}$} & \multirow{2}{*}{$\begin{array}{l}(\%) \\
\text { Hum. } \\
\text { Grano }\end{array}$} & \multirow{2}{*}{$\begin{array}{l}\text { Long. } \\
\text { (cm) }\end{array}$} & \multirow{2}{*}{$\begin{array}{c}\text { Diam. } \\
(\mathbf{c m})\end{array}$} & \multirow[t]{2}{*}{ Hiler. } \\
\hline & & & & & & & Tallo & Raíz & & & & & \\
\hline \multirow[t]{2}{*}{ Coataxtla } & $1 \times 24$ & Pob.21 x Pob.21 & 10,30 & 128,0 & 59 & 121 & 0,0 & 3,9 & 0,0 & 26,1 & 15,9 & 4,9 & 14 \\
\hline & $4 \times 24$ & Pob. $21 * 43 \times$ Pob. 32 & 10,01 & 124,3 & 58 & 129 & 2,5 & 2,5 & 0,0 & 27,0 & 17,1 & 5,2 & 16 \\
\hline \multirow[t]{2}{*}{ Poza Rica } & $9 \times 19$ & Pob.21 x Pob. 25 & 9,7 & 129,5 & 57 & 135 & 0,0 & 0,0 & 2,2 & 22,9 & 15,2 & 4,8 & 18 \\
\hline & L7x 14 & Pob43*43 x Pob. 24 & 9,58 & 127,9 & 56 & 133 & 8,0 & 0,0 & 3,5 & 19,1 & 15,4 & 5,0 & 18 \\
\hline \multirow[t]{2}{*}{ Cardel } & $5 \times 19$ & Pob. $21 * 21 \times$ Pob. 25 & 7,62 & 138,3 & - & 126 & 8,8 & 4,8 & 0,0 & 25,3 & 17,2 & 4,7 & 16 \\
\hline & $9 \times 19$ & Pob.21 x Pob. 25 & 7,00 & 127,0 & - & 124 & 8,8 & 4,2 & 1,0 & 26,4 & 15,2 & 4,8 & 18 \\
\hline \multirow[t]{2}{*}{ Tlaltizapan } & $4 \times 16$ & Pob. $21 * 43 \times$ Tuxp.Seq & 12,3 & 138,0 & 63 & 128 & 0,0 & 0,0 & 0,0 & 26,5 & 18,0 & 4,7 & 16 \\
\hline & $4 \times 24$ & Pob. $21 * 43 \times$ Pob. 21 & 11,5 & 129,1 & 64 & 133 & 2,4 & 1,2 & 1,2 & 22,9 & 17,1 & 5,2 & 16 \\
\hline \multirow[t]{2}{*}{ Gómez Farias } & $9 \times 24$ & Pob.21 x Pob.21 & 7,44 & 165,7 & 81 & 146 & 0,0 & - & 0,0 & 21,9 & 14,6 & 3,9 & 16 \\
\hline & $9 \times 22$ & Pob. 21 x Pob. 32 & 6,04 & 134,5 & 82 & 120 & 0,0 & - & 0,0 & 22,7 & 16,6 & 4,7 & 16 \\
\hline
\end{tabular}

1 Superioridad sobra la media general.

Con la información obtenida se muestra la importancia de considerar durante el desarrollo de líneas caracteres contrastantes en tipo de mazorca, así como su patrón heterótico para asegurar el éxito de un programa de hibridación.

\section{LITERATURA CITADA}

CIMMYT, 1994. World maize and trends. Maize seed industries, Revisted: Emergin Roles of the Public and Private Sectors. México, D.F. CIMMYT. pp. 1-23.
KEMPTHORNE, O. 1957. An introduction to genetics stadistic. New York: John Wiley and Sons, Inc: London Chapman, Hall, Ltd. pp. 468-470.

PANOEY, S; VARGAS, E. S. 1985. La interacción genotipomedio ambiente y su importancia en el mejoramiento intrapoblacional en las plantas cultivadas. VII Congreso Latinoamericano de Genética. I Congreso Colombiano de Genética, Bogotá, Colombia. p. 38.

SINGH, R.K; CHOUOHARY, B.O. 1985. Biometrical methods in quantitative genetics analysis. KALYANI publishers, India. 2a. ed. p..205-214. 
SRINIVASAN, G.; VASAL, S. K; GONZALEZ, F.; CORDOVA, H.; PANDEY, S.; VERGARA, N. 1993. Rendimiento y estabilidad de híbridos de maíz de cruzas simples evaluadas en Colombia, México y Centroamérica. Agron. Mesoam. 4: 23-29.

VASAL, S. K; BECK, D. L.; CROSSA, 1; VERGARA, N. 1986. Heterosis y aptitud combinatoria en germoplasma tropical de CIMMYT. XXXIII Reunión Anual del PCCMCA, Guatemala, Guatemala. p. 31-57.
VASAL, S. K; HAN, G.; ELIAS, E.; VERGARA, Y. N. 1990. Desarrollo de híbridos no convencionales de maíz. XIII Reunión de Maiceros de la Zona Andina, Chiclayo, Perú. p. 377-389.

VASAL, S. K.; GONZÁLEZ, F.; VERGARA, N.; SRINIVASAN, G. 1993. Comportamiento de híbridos intra e interpoblacionales entre líneas endocriadas e implicación de estos resultados en el desarrollo de híbridos de maíz. Agron. Mesoam. 4: 1-6. 
